\title{
Smooth Rational Curves on Enriques Surfaces
}

\author{
F. Cossec and I. Dolgachev * \\ Department of Mathematics, University of Michigan, Ann Arbor, MI 48109-1003, USA
}

\section{Introduction}

Let $S$ be an Enriques surface over an algebraically closed field $k$ of arbitrary characteristic $p$. Recall that this means that $S$ is a connected smooth projective surface whose canonical class is numerically trivial and second Betti number equal to 10 [4]. It is well-known that, generically over $k=\mathbb{C}$, an Enriques surface does not contain nonsingular rational curves. This can be seen, for example, by considering the period space for such surfaces [3]. Also, it is known that if $S$ contains such a curve, then, again generically, it contains infinitely many of them. This can be seen, for example, by viewing an Enriques surface as an elliptic surface whose jacobian surface is a rational elliptic surface. Assuming that the latter is general enough, its translation group is infinite and acts on $S$ by automorphisms. Thus, the existence of one such curve implies the existence of infinitely many. In this paper we prove the following rather surprising result:

Theorem. Let $S$ be an Enriques surface of degree d in a projective space $\mathbb{P}^{n}$. Assume that $S$ contains a smooth rational curve, then it contains such a curve of degree less or equal to $d$.

This result (Theorem 2.5 and its corollary) immediately implies that the subset of the Hilbert scheme parametrizing Enriques surfaces of degree $d$ in $\mathbb{P}^{n}$ containing smooth rational curves is a constructible subset. In fact, we prove a stronger result: this set is closed and its complement is dense if we assume that $\operatorname{char}(k) \neq 2$ (Theorems 3.4 and 3.6).

The result of the theorem above does not give the best estimate of the minimal degree of a smooth rational curve on a polarized Enriques surface. For example, by other means, we prove that an Enriques surface of degree 10 in $\mathbb{P}^{5}$ with a smooth rational curve must contain such a curve of degree less or equal to 4 . In one case this result was known: there exist a 9-parameter family of Enriques surfaces parametrizing lines in $\mathbb{P}^{3}$ included in at least two quadrics from a web of quadrics in $\mathbb{P}^{3}$ (Reye congruences). Every surface from this family embeds into $\mathbb{P}^{5}$ by

* The work of this author was partially supported by a NSF grant 
Plucker coordinates as a surface of degree 10 and contains smooth rational curves of degree less or equal to 4 .

The main technical tool in the proof of Theorem 2.5 is a lattice-theoretical result on the lattice $T_{2,3,7}$ isomorphic to the Neron-Severi lattice of an Enriques surface (Theorem 1.5).

All the results of these paper have analogues in the case where $S$ is a Coble rational surface, a surface obtained by blowing up 10 points on $\mathbb{P}^{2}$ which occur as the nodes of a rational irreducible plane curve of degree 6 . In fact, the study of these surface $[6,7]$ was one of the main sources of the ideas for this paper.

\section{A Lattice-Theoretical Result}

Here, by a lattice we mean an integral quadratic form, i.e. a free $\mathbb{Z}$-module $L$ equipped with a symmetric bilinear form $L \times L \rightarrow \mathbb{Z}$. The value of this form on a pair $(x, y)$ will be denoted by $x \cdot y$.

We denote by $\oplus$ the orthogonal sum of two lattices and by $L_{0}$ the set of isotropic vectors in $L$ (i.e. vectors $x \in L$ such that $x^{2}:=x \cdot x=0$ ).

We will be concerned with the two special lattices

$$
L=T_{2,3,7} \text { or } T_{2,4,5} \text {. }
$$

Recall the definition of the lattices $T_{p, q, r}$, where $p, q, r$ are arbitrary integers $\geqq 2$

where

$$
\begin{gathered}
T_{p, q, r}=\mathbb{Z} \alpha_{0} \oplus \mathbb{Z} \alpha_{1} \oplus \ldots \oplus \mathbb{Z} \alpha_{n-1}, \quad n=p+q+r-2, \\
\alpha_{i}^{2}=-2, \alpha_{i} \cdot \alpha_{j}=1 \text { or } 0
\end{gathered}
$$

according to whether $\alpha_{i}$ is joined to $\alpha_{j}$ or not in the following graph

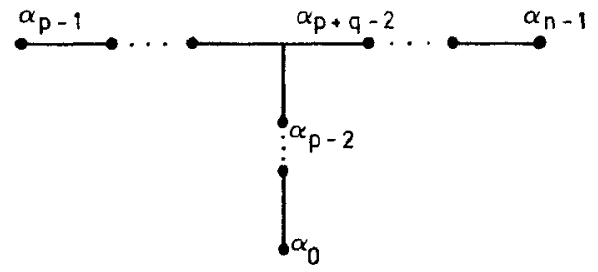

Let

$$
\bar{L}=\mathbb{Z} e_{0} \oplus \mathbb{Z} e_{1} \oplus \ldots \oplus \mathbb{Z} e_{n},
$$

where

$$
e_{0}^{2}=q-2, e_{1}^{2}=\ldots=e_{n}^{2}=-1 .
$$

Then, the lattice $T_{2, q, r}$ can be identified with the sublattice of $\bar{L}$ of all vectors orthogonal to the vector

$$
K=-q e_{0}+(q-2)\left(e_{1}+\ldots+e_{n}\right) .
$$

To see this it suffices to consider the vectors

$$
\alpha_{0}=e_{0}-e_{1}-\ldots-e_{q}, \alpha_{i}=e_{i}-e_{i+1}, \quad i=1, \ldots, n=q+r-1
$$


and to check that all of them are orthogonal to $K$ and form a basis in the orthogonal complement of $K$.

From now on $L=T_{2, q, r}$ with $(q, r)=(3,7)$ or $(4,5)$.

Lemma 1.1. Let $E_{7}(-1)$ and $E_{8}(-1)$ denote the lattices $T_{2,4,3}$ and $T_{2,3,5}$ respectively and $U=\mathbb{Z} v_{1} \oplus \mathbb{Z} v_{2}$, where $v_{1}^{2}=v_{2}^{2}=0$ and $v_{1} \cdot v_{2}=1$. Then

$$
\begin{aligned}
& T_{2,3,7} \cong U \oplus E_{8}(-1) \\
& T_{2,4,5} \cong U \oplus E_{7}(-1) .
\end{aligned}
$$

Proof. Clearly, the lattices $T_{2,4,3}$ and $T_{2,3,5}$ can be identified with the sublattices of $T_{2,4,5}$ and $T_{2,3,7}$ respectively which are spanned by the vectors $\alpha_{0}, \ldots, \alpha_{k}(k=6$ or $7)$.

$$
\begin{aligned}
& \text { Let } \\
& \qquad \begin{array}{ll}
f=2 \alpha_{0}+2 \alpha_{1}+2 \alpha_{2}+3 \alpha_{3}+4 \alpha_{4}+3 \alpha_{5}+2 \alpha_{6}+\alpha_{7}, & \text { if } L=T_{2,4,5} \\
f=3 \alpha_{0}+2 \alpha_{1}+4 \alpha_{2}+6 \alpha_{3}+5 \alpha_{4}+4 \alpha_{5}+3 \alpha_{6}+2 \alpha_{7}+\alpha_{8}, & \text { if } L=T_{2,3,7} .
\end{array}
\end{aligned}
$$

Then, we can identify the lattice $U$ with the sublattice of $L$ spanned by the vectors $f, f+\alpha_{n-1}$ and verify the lemma.

For every lattice $L$ and integer $m$ we let

$$
L_{m}=\left\{x \in L: x^{2}=-m\right\} \text {. }
$$

In particular, $L_{0}$ is the set of isotropic vectors in $L$ as above.

We denote the set $L_{2}$ by $R(L)$ (or simply $R$ if no confusion arise) and call the elements of $R(L)$ roots in $L$.

Let $W(L)$ be the subgroup of the orthogonal group $O(L)$ of the lattice $L$ which is generated by the transformations

$$
s_{\alpha}: x \rightarrow x+(x \cdot \alpha) \alpha,
$$

where $\alpha \in R(L)$ (called reflections). We call $W(L)$ the reflection (or Weyl) group of $L$. Lemma 1.2. Let $L=T_{p, q, r}$, where $p^{-1}+q^{-1}+r^{-1} \geqq 1$ or $(p, q, r)=(2,3,7),(2,4,5)$, $(3,3,4)$. Then

(i) $W(L) \cdot R(L)=W(L) \cdot \alpha$, for any $\alpha \in R(L)$.

(ii) $W(L)$ is a Coxeter group with respect to the set of generators

$$
S=\left\{s_{\alpha_{0}}, \ldots, s_{\alpha_{n-1}}\right\} .
$$

(iii) $W(L)$ is a normal subgroup of finite index in $O(L)$.

(iv) Let

$$
\left[O(L)=W(L) x\{ \pm 1\} \quad \text { for } L=T_{2,3,7} \text { or } T_{2,4,5}\right]
$$

$$
C=\left\{x \in L_{\mathbf{R}}=L \otimes \mathbb{R}: x \cdot x>0, x \cdot \alpha_{i} \geqq 0, \quad i=0, \ldots, n-1\right\} .
$$

Then for any $x \in L_{\mathbb{R}}\left(x^{2} \geqq 0\right.$ if $\left.(p, q, r)=(2,3,7),(2,4,5),(3,3,4)\right)$

$$
o(L) \cdot x \cap C \neq \emptyset \text {. }
$$

Proof. This is well-known. In the case $p^{-1}+q^{-1}+r^{-1}>1$, the lattice $L$ can be identified with the root lattice of a simple root system of type $D_{n}, E_{6}, E_{7}$, or $E_{8}$ 
equipped with the quadratic form given by the negative of the corresponding Cartan matrix. Then, all the assertions can be found in [5] [use the tables to check (i)].

In the case $p^{-1}+q^{-1}+r^{-1}=1$, i.e. $(p, q, r)=(2,4,4),(2,3,6)$ or $(3,3,3)$, the lattice $L \cong L^{\prime} \oplus \mathbb{Z} f$, where $L^{\prime}=T_{2,3,4}, T_{2,3,5}$, and $T_{2,3,3}$ respectively and $f^{2}=0(f$ can be taken as in the proof of Lemma 1.1 in the first two cases and $f=\alpha_{0}+2 \alpha_{1}$ $+\alpha_{2}+2 \alpha_{3}+3 \alpha_{4}+2 \alpha_{5}+\alpha_{6}$ in the case $\left.T_{3,3,3}\right)$. It is easy to see that $W(L)$ coincides with the affine Weyl group $W_{a}$, where $W=W(L)$ [5, Chap. 6, Sect. 2]. All the assertions can be found again in [5].

The cases $(p, q, r)=(2,3,7),(2,4,5)$, and $(3,3,4)$ are more delicate. The groups $W(L)$ are crystallographic reflection groups in a Lobachevski space. All the assertions are verified by constructing the corresponding fundamental polyhedron following Vinberg's algorithm [18].

Corollary 1.3. Let $\propto$ be a root in $T_{2,3,7}$. Then the orthogonal complement of the sublattice $\mathbb{Z} \alpha$ is isomorphic to the lattice $T_{2,4,5}$.

Proof. The lattice $T_{2,4,5}$ embeds naturally into $T_{2,3,7}$ by embedding $E_{7}(-1)$ into $E_{8}(-1)$ and using Lemma 1.1. Its orthogonal complement in $T_{2,3,7}$ is a lattice of rank 1 and discriminant -2 . Thus, $T_{\mathbf{2}, \mathbf{4}, 5}$ is the orthogonal complement of $\mathbb{Z} \alpha$ for some root $\alpha$. The result follows by applying Lemma 1.2(i).

Lemma 1.4. Let $L=T_{2,3,7}$. Define

$$
\begin{aligned}
& f_{i}=-K+e_{i}, \quad i=1, \ldots, 10 ; \\
& \Delta=-3 K+e_{0}=\left(f_{1}+\ldots+f_{10}\right) / 3 .
\end{aligned}
$$

Then the above vectors belong to $L$ and satisfy

$$
f_{i}^{2}=0, f_{i} \cdot f_{j}=1, i \neq j, f_{i} \cdot \Delta=3, \Delta^{2}=10 \text {. }
$$

Moreover, the vectors

$$
w_{0}=\Delta, w_{1}=\Delta-f_{1}, w_{2}=2 \Delta-f_{1}-f_{2}, w_{i}=f_{i+1}+\ldots+f_{10}, \quad i=3, \ldots, 10
$$

form the dual basis to the root basis $\alpha_{0}, \ldots, \alpha_{9}$ in $L^{*}=L$.

Proof. Direct verification.

Let $L$ be any indefinite lattice. Following [8] we can introduce the function

$$
\phi_{L}: L \rightarrow \mathbb{Z}_{\geqq 0}
$$

by putting

$$
\phi_{L}(x)=\min _{f \in L_{0}-\{0\}}|x \cdot f| .
$$

Obviously, this function is constant on $O(L)$-orbits. If $L$ is hyperbolic [i.e. $\left.\operatorname{sign}\left(L_{\mathrm{R}}\right)=(1, \operatorname{rk}(L)-1)\right]$, then $\phi_{L}(x)>0$ for every $x$ with $x^{2}>0$.

Also, for every $\alpha \in L_{2}$ we define $\phi_{\alpha}: L \rightarrow \mathbb{Z}_{\geqq 0}$ by

$$
\phi_{a}(x)=\min _{\substack{f \in L_{0}-\{0\} \\ f \cdot x=0}}|x \cdot f| .
$$


Theorem 1.5. Let $\alpha$ be a root in $L=T_{2,3,7}$ and $x \in L$ with $x \cdot x>0$. Then

$$
\phi_{a}(x)<x \cdot \alpha \quad \text { or } x \cdot \alpha \leqq \frac{3}{\phi_{L}(x)} x^{2} .
$$

Proof. Obviously, we may assume that $x \cdot \alpha>0$. By Corollary 1.3, we know that $L_{\alpha}$ is isomorphic to the lattice $T_{2,4,5}$. Without loss of generality, we may assume that $\alpha=\alpha_{9}$. Then an explicit root basis in $L_{\alpha}$ can be given by

$$
\begin{gathered}
\alpha_{0}^{\prime}=\alpha_{0}, \alpha_{1}^{\prime}=e_{0}-e_{1}-e_{9}-e_{10}=\alpha_{0}+\alpha_{2}+2 \alpha_{3}+2 \alpha_{4}+2 \alpha_{5}+2 \alpha_{6}+2 \alpha_{7}+2 \alpha_{8}+\alpha_{9}, \\
\alpha_{i}^{\prime}=\alpha_{i}, \quad i=2, \ldots, 8 .
\end{gathered}
$$

Its dual basis in $L_{\alpha}^{*} \subset \frac{1}{2} L_{\alpha}$ is $\left\{\omega_{0}^{\prime}, \ldots, \omega_{8}^{\prime}\right\}$, where $\omega_{0}^{\prime}=\omega_{0}-\frac{1}{2} \omega_{8}, \omega_{1}^{\prime}=\frac{1}{2} \omega_{8}, \omega_{2}^{\prime}=\omega_{1}, \omega_{3}^{\prime}=\omega_{2}-\frac{1}{2} \omega_{8}, \omega_{i}^{\prime}=\omega_{i-1}-\omega_{8}, \quad i=4, \ldots, 8$.

Let $y$ be a vector from $L_{\alpha}$ defined by

$$
2 x+(x \cdot \alpha) \alpha=y \text {. }
$$

Note that $y \cdot y=4 x \cdot x+2(x \cdot \alpha)^{2}>0$.

Applying transformations from the Weyl group of $L_{\alpha}$, we may assume that $y$ belongs to the set $C^{\prime}=\mathbb{R}_{\geqq 0} \omega_{0}^{\prime}+\ldots+\mathbb{R}_{\geqq 0} \omega_{\mathbf{8}}^{\prime}$ (a fundamental chamber), that is, $y$ can be written in the form

$$
y=a_{0} \omega_{0}^{\prime}+\ldots+a_{8} \omega_{8}^{\prime},
$$

where $a_{i}$ are nonnegative integers.

Next, recalling the notation of Lemma 1.4, we observe that

$$
\begin{aligned}
\omega_{0}^{\prime}= & \left(\omega_{0}^{\prime}-\frac{3}{2} \alpha\right)+\frac{3}{2} \alpha=\left(\Delta-\frac{1}{2} f_{9}-\frac{1}{2} f_{10}-\frac{3}{2} f_{9}+\frac{3}{2} f_{10}\right)+\frac{3}{2} \alpha \\
= & \left(\Delta-2 f_{9}+f_{10}\right)+\frac{3}{2} \alpha=g_{0}+\frac{3}{2} \alpha, \\
\omega_{1}^{\prime}= & \left(\frac{1}{2} \omega_{8}-\frac{1}{2} \alpha\right)+\frac{1}{2} \alpha=\frac{1}{2}\left(f_{9}+f_{10}\right)-\frac{1}{2}\left(f_{9}-f_{10}\right)+\frac{1}{2} \alpha \\
= & f_{10}+\frac{1}{2} \alpha=g_{1}+\frac{1}{2} \alpha, \\
\omega_{2}^{\prime}= & \left(\omega_{1}-\alpha\right)+\alpha=\left(\Delta-f_{1}-f_{9}\right)+f_{10}+\alpha=g_{2}+g_{1}+\alpha, \\
\omega_{3}^{\prime}= & 2 \Delta-f_{1}-f_{2}-\frac{1}{2}\left(f_{9}+f_{10}\right)=f_{10}+\left(\Delta-f_{1}-f_{9}\right)+\left(\Delta-f_{2}-f_{9}\right) \\
& +\frac{3}{2}\left(f_{9}-f_{10}\right)=g_{1}+g_{2}+g_{3}+\frac{3}{2} \alpha, \\
\omega_{4}^{\prime}= & \left(f_{4}+\ldots+f_{10}\right)-\left(f_{9}+f_{10}\right)=f_{4}+\ldots+f_{8} \\
= & \left(f_{4}+f_{5}-\alpha\right)+\left(f_{6}+f_{7}-\alpha\right)+f_{8}+2 \alpha=g_{4}+g_{5}+g_{6}+2 \alpha, \\
\omega_{5}^{\prime}= & f_{5}+\ldots+f_{8}=\left(f_{5}+f_{6}-\alpha\right)+\left(f_{7}+f_{8}-\alpha\right)+2 \alpha=g_{7}+g_{8}+2 \alpha, \\
\omega_{6}^{\prime}= & f_{6}+f_{7}+f_{8}=\left(f_{6}+f_{7}-\alpha\right)+f_{8}=g_{5}+g_{6}+\alpha, \\
\omega_{7}^{\prime}= & f_{7}+f_{8}=\left(f_{7}+f_{8}-\alpha\right)+\alpha=g_{8}+\alpha, \\
\omega_{8}^{\prime}= & f_{8}=g_{6},
\end{aligned}
$$

where $g_{0}, \ldots, g_{8}$ are isotropic vectors in $L$. 
Plugging these expressions in, we obtain

$$
y=b_{0} g_{0}+\ldots+b_{9} g_{9}+b \alpha,
$$

where all $b_{i}$ and $b$ are nonnegative integers.

Since $y \cdot \alpha=0$, we have

$$
2 b=3 b_{0}+b_{1}+b_{2}+b_{3}+2 b_{4}+2 b_{5}+2 b_{7}+2 b_{8} .
$$

Assume that $x \cdot \alpha>b$. Then,

$$
2 \phi_{\alpha}(x)=\phi_{a}(y) \leqq y \cdot f_{8}=2 b_{0}+b_{1}+b_{2}+b_{3}+2 b_{4}+2 b_{5}+2 b_{7}+b_{8}=2 b-b_{0}-b_{8} .
$$

Thus, in this case

$$
\phi_{\alpha}(x)<x \cdot \alpha
$$

Assume that $x \cdot \alpha \leqq b$. Then,

$$
2 x=\sum b_{i} g_{i}+(b-(x \cdot \alpha)) \alpha,
$$

hence,

$$
\begin{aligned}
2 x^{2} & =\sum_{i} b_{i}\left(g_{i} \cdot x\right)+(b-(x \cdot \alpha))(x \cdot \alpha) \geqq \phi_{L}(x) \sum_{i} b_{i}, \\
2 x \cdot \alpha & =\sum_{i} b_{i}\left(g_{i} \cdot \alpha\right)-2(b-(x \cdot \alpha)) \leqq 3 \sum_{i} b_{i} .
\end{aligned}
$$

This, obviously, proves what we want.

Remark 1.6. For an indefinite lattice $L$ it would be interesting to find an estimate of the function $\phi_{L}$. It was proven by E. Looijenga (unpublished) that

$$
\phi_{L}(x)^{2} \leqq a x^{2},
$$

where $a=1,2,3 / 2$ for the lattices $T_{2,3,7}, T_{2,4,5}, T_{3,3,4}$ respectively. Applying this result to the vector $y=2 x+(x \cdot \alpha) \alpha$ from the proof of the previous theorem, we obtain

$$
4 \phi_{a}(x)^{2}=\phi_{\alpha}(y)^{2}=\phi_{T_{2,4,5}}(y)^{2} \leqq 2 y^{2}=8 x^{2}+4(x \cdot \alpha)^{2},
$$

that is,

$$
\phi_{\alpha}(x)^{2} \leqq 2 x^{2}+(x \cdot \alpha)^{2} .
$$

This is rather close to our result, but, unfortunately, is not enough for the applications of the next section.

\section{Rational Curves on an Enriques Surface}

Recall [4] that an Enriques surface is a nonsingular projective surface $S$ such that

$$
K_{S} \equiv 0, B_{2}(S)=\operatorname{dim} H_{e t}^{2}\left(S, Q_{\ell}\right)=10 \text {. }
$$

If $\operatorname{char}(k)=p \neq 2$, then the above definition is equivalent to the classical one:

$$
K_{s} \neq 0,2 K_{s}=0, H^{1}\left(S, \mathcal{O}_{s}\right)=0 \text {. }
$$


Let $\operatorname{Pic}(S)$ be the Picard group of $S$ and

$$
H_{S}=\operatorname{Pic}(S) / \text { numerical equivalence. }
$$

The intersection form on $\operatorname{Pic}(F)$ equips $H_{S}$ with a lattice structure.

Lemma 2.1 .

$$
H_{S} \cong T_{2,3,7} \text {. }
$$

Proof. See [13, Theorem 0.4] and apply Lemma 1.1 (cf. also [10, 2.1]).

For every divisor $D$ on $S$ we denote by $[D]$ its class in $H_{S^{-}}$. Let $H_{S}^{+}$denote the set of classes of effective divisors on $S$.

Lemma 2.2. Let $h \in H_{S}$ be the class of an ample divisor on $S$. Let $x \in H_{S}$ be such that $x^{2} \geqq 0$ and $x \cdot h>0$. Then $x \in H_{S}^{+}$.

Proof. Let $x=[D]$. By Riemann-Roch

$$
h^{0}(D)+h^{0}\left(K_{S}-D\right) \geqq \frac{1}{2} D^{2}+\chi\left(\mathcal{O}_{S}\right) .
$$

It is known $[4, \mathrm{p} .25]$ that $\chi\left(\mathcal{O}_{S}\right)=1$. Thus, $[D]$ or $[-D]$ belongs to $H_{S}^{+}$. The assumption $x \cdot h>0$ implies $x=[D] \in H_{S}^{+}$.

Let $R_{S}^{+}$be the set of the classes $[E]$ of nonsingular rational curves $E$ on $S$. Since $K_{S} \equiv 0, E^{2}=-2$ and, hence,

$$
R_{S}^{+} \subset R\left(H_{S}\right) \text {. }
$$

Definition. An Enriques surface $S$ is called nodal (resp. unnodal) if $R_{S}^{+} \neq \emptyset$ (resp. $R_{S}^{+}=\emptyset$ ).

Lemma 2.3. An Enriques surface $S$ is nodal if and only if

$$
R\left(H_{S}\right) \cap H_{S}^{+} \neq \emptyset \text {. }
$$

Proof. Obviously, $R_{S}^{+} \subset H_{S}^{+}$. Conversely, assume that $x \in H_{S}^{+} \cap R\left(H_{S}\right)$. Write $x=[D]$ for some effective divisor $D=\sum n_{i} E_{i}$, where $E_{i}$ are its irreducible components. Clearly, $E_{i}^{2} \geqq-2$. Since $D^{2}=-2$, we must have $E_{i}^{2}=-2$ for at least one component $E_{i}$ of $D$. By the genus formula, $E_{i}$ is a nonsingular rational curve. Hence, $S$ is nodal.

Since $|E|=\{E\}$ for every nonsingular rational curve $E$ on $S$, we may identify the set of such curves with the set $R_{S}^{+}$.

Recall that an elliptic(resp. a quasi-elliptic) pencil on $S$ is a morphism $f: S \rightarrow \mathbb{P}^{1}$ whose general fibre is a smooth elliptic curve (resp. geometrically irreducible curve of arithmetical genus 1).

Lemma 2.4. Let $x \in\left(H_{S}\right)_{0} \cap H_{S}^{+}$. Assume that $x$ is primitive (i.e. is not divisible by any integer $m>1$ ) and $x \cdot e \geqq 0$ for all $e \in R_{S}^{+}$. Then $2 x$ is the class of a fibre of an elliptic or a quasi-elliptic fibration on $S$. Conversely, the class of such a fibre is equal to $2 x$, where $x$ is a primitive isotropic vector in $H_{S}$.

Proof. This is well-known (see, for example, [13]).

Now we can prove the main result of this paper. 
Theorem 2.5. Let $S$ be a nodal Enriques surface and $H$ be an ample divisor on $S$. Then, there exists a smooth rational curve on $S$ such that

$$
E \cdot H \leqq 3 H^{2} / \phi_{H_{S}}([H]) \text {. }
$$

Proof. Let $\alpha_{0} \in R_{S}^{+}$be the class of a smooth rational curve on $S$ and $h=[H]$ be the class of $H$. Assume that

$$
\alpha_{0} \cdot h>3 h^{2} / \phi_{H_{S}}(h) .
$$

Then, it follows from Theorem 1.5 that one can find an isotropic vector $f$ in $H_{S}$ such that

$$
0<f \cdot h<h \cdot \alpha_{0}, \quad f \cdot \alpha_{0}=0 .
$$

Clearly, we may assume that $f$ is primitive. Let $\alpha_{0}=\left[E_{0}\right]$, where $E_{0}$ is a nonsingular rational curve. By Lemma $2.2, f=[D]$ for some positive divisor $D$. Since $f \cdot h<h \cdot \alpha_{0}, E_{0}$ is not a component of $D$. Since $D \cdot E_{0}=0, E_{0}$ does not intersect any irreducible component of $D$. In particular, for every $e \in R_{S}^{+}$such that $f \cdot e<0$, we must have $e \cdot \alpha_{0}=0$. Assume that $f \cdot e<0$ for some $e \in R_{S}^{+}$. Then

$$
f^{\prime}=s_{e}(f)=f+(f \cdot e) e
$$

satisfies

$$
f^{\prime} \cdot \alpha_{0}=0, f^{\prime} \cdot h<f \cdot h .
$$

Also, $f^{\prime} \in H_{S}^{+}$, otherwise, by Lemma 2.2

$$
-f^{\prime}=-(f \cdot e) e-f
$$

is effective. This contradicts the obvious fact that $|m E|$ is isolated for any nonsingular rational curve $E$ on $S$ and $m>0$. Thus, replacing $f$ by $f^{\prime}$, we may assume that $f \cdot e \geqq 0$ for all $e \in R_{S}^{+}$. Applying Lemma 2.4 , we obtain that $|2 D|$ defines an elliptic or a quasi-elliptic pencil on $S$. Since $E_{0} \cdot D=0, E_{0}$ must be an irreducible component of a member of $|2 D|$. Let $E_{1}$ be another component of the same fibre (necessarily a nonsingular rational curve). We have

$$
h \cdot E_{1} \leqq\left[2 D-E_{0}\right] \cdot h=2 f \cdot h-h \cdot \alpha_{0}<h \cdot \alpha_{0} .
$$

Proceeding in this way, we find a nonsingular rational curve satisfying the inequality.

Remark 2.6. Notice that typically a nodal Enriques surface has infinitely many nonsingular rational curves. If $k=\mathbb{C}$, then a nodal Enriques surface has finitely many nonsingular rational curves if and only if its automorphism group is finite [15]. Notice that the latter happens very rarely. In fact, all Enriques surfaces with finite automorphism group have been explicitly classified recently by S. Kondō.

Corollary 2.7. In the notation of Theorem 2.5, assume that $H$ is a very ample divisor. Then there exists a nonsingular rational curve $E$ on $S$ such that

$$
E \cdot H \leqq H^{2} \text {. }
$$


Proof. It suffices to show that $\phi_{H_{S}}(H) \geqq 3$. Let $f$ be a primitive isotropic vector in $H_{S}$. We may assume that $f=[D]$, where $D$ is a positive divisor. Write $D=\sum n_{i} C_{i}$, where $C_{i}$ are irreducible. Since $H$ is very ample, $H \cdot C_{i}>0$ for all $i$. Thus, either $D \cdot H \geqq 3$, or $D=C_{1}+C_{2}$, where $C_{1} \cdot H=C_{2} \cdot H=1$, or $D$ is irreducible, $D \cdot H \leqq 2$. The linear system $|H|$ embeds $S$ into a projective space, and the image of $D$ is a curve of degree $D \cdot H$. In the first case, we obtain that $D$ is the union of two lines, hence $C_{1} \cdot C_{2} \leqq 1$. This is impossible, because $D^{2}=C_{1}^{2}+C_{2}^{2}+2 C_{1} \cdot C_{2} \leqq-2$. In the second case, $D$ is a conic or a line, again this is impossible.

Remark 2.8. With substantially more effort one can show that the assertion of the corollary is still true for any ample divisor $H$.

\section{Polarized Enriques Surfaces}

Let $S$ be an Enriques surface embedded into a projective space $\mathbb{P}^{n}$. If $\operatorname{deg}(S)=d$, then the Hilbert polynomial

$$
P_{S}(m)=\chi\left(\mathcal{O}_{S}(m)\right)=\frac{1}{2} m^{2} d+1 .
$$

Lemma 3.1. Let $S$ be a nonsingular connected surface of degree $d$ in $\mathbb{P}^{n}$ with the Hilbert polynomial $P_{S}(m)=\frac{1}{2} m^{2} d+1$. Then $S$ is an Enriques surface or a rational surface.
Proof. We have
a) $K_{S} \cdot H=0$,
b) $\chi\left(\mathcal{O}_{s}\right)=1$,

where $H$ is a hyperplane section of $S$.

It follows from a) that either $K_{S}$ is numerically trivial, or $\left|m K_{S}\right|=\emptyset$ for all integers $m \neq 0$. In the second case, together with $b$ ), we get by Castelnuovo's criterion that $S$ is rational. Suppose the first case occurs. Then, $p_{g}(S)=0$ or $p_{g}(S)=1$ and $K_{S}=0$. It follows from b) that $h^{1}\left(\mathcal{O}_{S}\right)=0$ or 1 respectively. If $h^{1}(S)=0$, then $B_{1}(S)=0$ and by Noether's formula $c_{2}(S)=12$. This shows that $B_{2}(S)=10$ and $S$ is an Enriques surface. If $h^{1}\left(\mathcal{O}_{S}\right)=1$, then we get similarly that $B_{2}(S)=10+2 B_{1}(S)$. If $B_{1}(S)=0$, then $S$ is an Enriques surface. If $B_{1}(S)=2$ [clearly, $B_{1}(S)=2 \operatorname{dim} A \operatorname{lb}(S)$ $\left.\leqq 2 h^{1}\left(\mathcal{O}_{S}\right)\right]$, then $B_{2}(S)=14$. However, Theorem 5 of [4] shows that no surfaces with $K_{S}=0, B_{2}(S)=14$ exist.

Remark 3.2. If $S$ is embedded in $\mathbb{P}^{n}$ by a complete linear system and $H^{1}\left(S, \mathcal{O}_{S}\right)=0$ [e.g. $\operatorname{char}(k) \neq 2$ ], then $\operatorname{deg}(S)=2 n$. This follows from Ramanujan's vanishing theorem [13, Theorem 0.8$]$.

Let Hilb $_{\mathbf{p}^{n}}^{P(m)}$ be the Hilbert scheme parameterizing surfaces in $\mathbb{P}^{n}$ with the Hilbert polynomial $P(m)=\frac{1}{2} m^{2} d+1$. Let $\mathbf{H}_{E}^{d}$ be the open subset of this Hilbert scheme parametrizing Enriques surfaces embedded into $\mathbb{P}^{n}$ by a complete linear system. By the previous Remark, $n=\frac{1}{2} d$ if $H^{1}\left(S, \mathcal{O}_{S}\right)=0$.

Lemma 3.3. Assume that $\operatorname{char}(k) \neq 2$. Then $\mathbf{H}_{E}^{d}$ is a smooth scheme of dimension $4 d^{2}+4 d+10$ at each of its points.

Proof. We know that

$$
\begin{gathered}
H^{0}\left(S, \Theta_{S}\right)=H^{2}\left(S, \Theta_{S}\right)=H^{2}\left(S, \mathcal{O}_{S}\right)=H^{1}\left(S, \mathcal{O}_{S}(1)\right)=0, \\
\operatorname{dim} H^{1}\left(S, \Theta_{S}\right)=10
\end{gathered}
$$


where $\Theta_{S}$ is the tangent sheaf of an Enriques surface $S \subset \mathbb{P}^{n}$ of degree $d$ [13]. Using this, the proof is standard [16, Sect. 2].

Theorem 3.4. Let ${ }^{n} \mathbf{H}_{E}^{d}$ be the subset of $\mathbf{H}_{E}^{d}$ parametrizing nodal Enriques surfaces. Then ${ }^{n} \mathbf{H}_{E}^{d}$ is a closed subset of $\mathbf{H}_{E}^{d}$.

Proof. By Theorem 2.5, we know that every Enriques surface of degree $d$ in $\mathbb{P}^{n}$ is either unnodal or contains a rational curve of degree $\leqq d$.

Let $p: \mathbf{X} \rightarrow \mathbf{H}_{E}^{d}$ be the universal family of Enriques surfaces over $\mathbf{H}_{E}^{d}$. For each positive integer $t \leqq d$, we may consider the Hilbert scheme

$$
\text { Hilb }_{X / H_{E}^{d}}^{P_{t}}
$$

where $P_{t}(m)=m t+1$. By Grothendieck, this is a quasi-projective scheme over $\mathbf{H}_{E^{\prime}}^{d}$ Its fibre over a geometric point $\bar{u}$ of $H_{E}^{\mathrm{d}}$ parametrizes nonsingular rational curves of degree $t$ lying on the Enriques surface $S_{\bar{u}}=p^{-1}(u) \underset{k(u)}{\bigotimes} \overline{k(u)}$. It follows from Chevalley's theorem that the set

$$
{ }^{n} \mathbf{H}_{E}^{d}=\left\{u \in \mathbf{H}_{E}^{d}:\left(\mathbf{H i l h}_{\mathbf{X} / \mathbf{H}_{\mathbf{E}}^{d}}^{P_{\bar{u}}} \neq \emptyset \text { for some } t<N\right\}\right.
$$

is a constructible subset of $\mathbf{H}_{E}^{d}$. To prove that it is a closed subset, it suffices to show this set is stable under specializations. Let $\eta \in{ }^{n} \mathrm{H}_{E}^{d}$, and $t \in \overline{\{\eta\}}$ be its specialization. Since $f$ is proper and smooth, we have a specialization homomorphism (SGA VI, Exp. X, 7.17.3.2):

$$
\text { sp: } H_{S_{\bar{\eta}}} \rightarrow H_{S_{\bar{t}}} \text {, }
$$

where $S_{\bar{\eta}}, S_{\bar{t}}$ are geometric fibres of $f$ over $\eta$ and $t$ respectively. It follows from the construction of $\mathrm{sp}$ that $\mathrm{sp}\left(H_{S_{\bar{\eta}}}^{+}\right) \subset H_{S_{\vec{\tau}}}^{+}$Also, it is known that sp is a homomorphism of the lattices. Thus, if $S_{\bar{\eta}}$ is nodal, $S_{\bar{\imath}}$ has an ${ }^{\circ}$ effective divisor $D$ with $D^{2}=-2$. Then, one of the irreducible components of $D$ is a nonsingular rational curve (Lemma 2.3), i.e. $S_{\bar{t}}$ is nodal. This proves the theorem.

Remark 3.5. In general, $\mathbf{H}_{E}^{\mathbf{d}}$ is not connected. The number of its connected components is related to the number of the orbits of vectors $x \in T_{2,3,7}$ with $x^{2}=d$ and $\phi(x) \geqq 3$ under the action of the Weyl group $W$.

We also do not know, whether ${ }^{n} \mathbf{H}_{E}^{d}$ is a proper subset of $\mathbf{H}_{E}^{d}$. We show below that this is true in the case $\operatorname{char}(k) \neq 2$.

Theorem 3.6. Assume char $(k) \neq 2$. Then

$$
{ }^{u n} \mathbf{H}_{E}^{d}=\mathbf{H}_{E}^{d}-{ }^{n} \mathbf{H}_{E}^{d}
$$

is an open dense subset of $\mathbf{H}_{\mathrm{E}}^{d}$.

Proof. Let $U$ be a connected component of $\mathbf{H}_{E}^{d}$ and ${ }^{n} U={ }^{n} \mathbf{H}_{E}^{d} \cap U$. By Theorem 3.5, it suffices to show that $U \neq{ }^{n} U$. Assume $U={ }^{n} U$. The restriction of $p: \mathbf{X} \rightarrow \mathbf{H}_{E}^{n}$ over $U$ is a deformation of one of its fibres, $X_{u_{0}}=S_{0}$. Since $H^{0}\left(S_{0}, \Theta_{S_{0}}\right)=H^{2}\left(\mathcal{O}_{S_{0}}\right)=0$ $(\operatorname{char}(k) \neq 2)$, we have a universal deformation space for $S_{0}$, pro-represented by

$$
D=\operatorname{Sp} f\left(k\left[\left[T_{1}, \ldots, T_{10}\right]\right]\right) .
$$

Since $H^{2}\left(S_{0}, \mathcal{O}_{S_{0}}\right)=0$, this deformation is effective, and by Artin's theorem [1] is algebraizable. Thus, there exists a smooth connected $k$-scheme $T$ of finite type, a 
smooth proper morphism $f: \mathbf{Y} \rightarrow T$ and a closed point $t_{0} \in T$ such that $\mathbf{Y}_{t_{0}}=S_{0}$ and $\mathrm{Y} \times \hat{\mathcal{O}}_{T, t_{0}} \rightarrow \operatorname{Spec} \hat{\mathcal{O}}_{T, t_{0}}$ ffectively pro-represents deformations of $S_{0}$.

Suppose that the generic geometric fibre $\mathbf{Y}_{\bar{\eta}}$ of $f$ is a nodal Enriques surface. By a Theorem 6.1 of [8], $Y_{\bar{\eta}}$ is of special type. This means that there exist a rational nonsingular curve $C_{\bar{\eta}}$ and an irreducible elliptic or a quasi-elliptic pencil $\left|F_{\bar{\eta}}\right|$ on $Y_{\bar{\eta}}$ such that $C_{\bar{\eta}} \cdot F_{\bar{\eta}}=2$.

Let us show that the curves $C_{\bar{\eta}}$ and $F_{\bar{\eta}}$ can be defined over a finite separable extension $K^{\prime}$ of the field $K=k(\eta)$. Indeed, lifting the very ample sheaf $\mathcal{O}_{S_{0}}(1)$ to $\mathbf{Y}$, we may assume that $f$ is a family of polarized Enriques surfaces in $\mathbb{P}^{n}$ (of course, we replace $T$ by an open neighborhood of $t_{0}$ if needed). Let $a=\operatorname{deg}\left(C_{\bar{\eta}}\right), b=\operatorname{deg}\left(F_{\bar{\eta}}\right)$, $P(m)=a m+1, P^{\prime}(m)=b m$, and

\section{$U \subset$ Hilb $_{\mathbf{Y} / T}^{P}, \quad U^{\prime} C \mathbf{H i l b}_{\mathbf{Y} / T}^{P^{\prime}}$}

be the open subsets of the corresponding Hilbert schemes whose geometric generic fibres contain the points associated to the curves $C_{\bar{\eta}}$ and $F_{\bar{\eta}}$ respectively. Since

$$
H^{0}\left(C, \mathscr{O}_{C}(C)\right)=0\left[\text { resp. } \operatorname{dim} H^{0}\left(F, \mathcal{O}_{F}(F)\right)=\operatorname{dim}|F|\right]
$$

for any rational nonsingular curve $C$ (resp. irreducible curve $F$ of arithmetic genus 1) on an Enriques surface $S$, the schemes $U$ and $U^{\prime}$ are smooth over $T$. In particular, $C_{\bar{\eta}}$ is defined over the residue field of a point of $U_{\eta}$ which must be a separable extension of $K$. Similarly, replacing $F$ by a linear equivalent curve, we may assume that $F$ is defined over a residue field of the smooth curve $U_{\eta}^{\prime}$ which is a separable extension of $K$.

Let $T^{\prime}$ be a normalization of $T$ in $K^{\prime}$. Since $K^{\prime}$ is a separable extension of $K, T^{\prime}$ is etale over a certain open neighborhood of $t_{0}$. Thus, we may replace $T$ by an etale neighborhood of $t_{0}$ to assume that $C_{\bar{\eta}}$ and $F_{\bar{\eta}}$ are defined over $k(\eta)$. Now, by a standard specialization argument, we can find an open subset $U$ of $T$ such that $C_{\bar{\eta}}$ and $F_{\bar{\eta}}$ extend to a family $\mathbf{C}$ and $\mathbf{F}$ over $U$ with $\mathbf{C}_{t}$ (resp. $\mathbf{F}_{t}$ ) a nonsingular rational curve (resp. $\mathbf{F}_{t}$ is an irreducible elliptic or a quasi-elliptic pencil) on $\mathbf{Y}_{t}, \mathbf{C}_{t} \circ \mathbf{F}_{t}=2$, for all $t \in U$. The pair $(\mathbf{C}, \mathbf{F})$ defines a structure of a family of $U$-marked Enriques surfaces on the family $f: Y_{U} \rightarrow U$ [10]. Replacing $U$ by a smaller set, we may assume that the system $|2 \mathbf{C}+\mathbf{F}|$ defines a morphism $g: Y_{U} \rightarrow \mathbb{P}_{U}^{4}$ which is generically $2: 1$ to its image and the ramification subscheme is a family $\mathbf{W} \rightarrow U$ of curves of arithmetical genus 5 with at most $a_{n}, d_{n}, e_{n}$-points as singularities which are canonically embedded into $\mathbb{P}^{4}$. Each curve $W_{u}$ from this family can be given as an intersection of three quadrics, two of them, up to a projective transformation can be chosen in the form:

$$
x_{0}^{2}+x_{1}^{2}+x_{2}^{2}=0, \quad x_{0} x_{4}+x_{3}^{2}=0 .
$$

The coarse moduli space of such curves is a 9-dimensional variety $\mathbf{M}$. The fibres of the canonical map $\phi: U \rightarrow \mathbf{M}$ represent isomorphic Enriques surfaces (loc. cit.). Since the property of formal versality is an open condition $[2,4.4]$, we can replace $U$ by a smaller open set to assume that $\mathbf{Y} \rightarrow \mathcal{O}_{T, t}$ is a versal deformation of $\mathbf{Y}_{t}$ for all closed points $t \in U$. However, the deformation of $\mathbf{Y}_{t}$ along the tangent vector to the fibre of the map $\phi: U \rightarrow \mathbf{M}$ is trivial. This contradiction shows that the generic geometric fibre $\mathbf{Y}_{\eta}$ of $f$ is an unnodal Enriques surface (cf. [13, p. 63], where this fact is proven in the case char $(k)=0)$. Now, since $\mathbf{Y} \times \hat{\mathcal{O}}_{T, t_{0}}$ is a versal deformation 
of $\mathbf{X}_{u_{0}}=S_{0}$, we obtain that the geometric generic fibre of $\mathbf{X} \times \hat{\mathcal{O}}_{U, u_{0}} \rightarrow \operatorname{Spec} \hat{\mathscr{O}}_{U, u_{0}}$ is an unnodal Enriques surface. Obviously, this implies that the geometric generic fibre of $\mathbf{X}_{U} \rightarrow U$ is an unnodal Enriques surface. Thus, ${ }^{n} \mathbf{H}_{E}^{d}$ is a proper closed subset in every connected component of $\mathbf{H}_{E}^{d}$ and the theorem is proven.

Remark 3.7. In the case $k=\mathbb{C}$ one can easily prove Theorem 3.6 by using the periods of Enriques surfaces [3,2.5]. In fact, one can prove a little more. Recall that by Horikawa's result [11], the isomorphism classes of Enriques surfaces are parametrized by a quasi-projective variety $\mathbf{M}_{E}=D^{0} / \Gamma$, where $D^{0}$ is the complement of a certain analytic set in the union of two copies of a bounded symmetric domain of dimension 10 and of type IV, and $\Gamma$ is a certain arithmetic group of automorphisms of $D^{0}$. As is explained in [3], the set of unnodal surfaces is parametrized by the set $D_{\text {gen }} / \Gamma$, where $D_{\text {gen }}=D^{0}-\bigcup_{c \in R(L)} D_{c^{\prime}}$. Here $L$ is the lattice $T_{2,3,7}$, and $D_{c}$ is a certain irreducible hypersurface in $D$. It was shown by Namikawa [15, Theorem 6.4] that all $D_{c}$ 's are $\Gamma$-equivalent and define an irreducible hypersurface ${ }^{n} \mathbf{M}_{E}$ in $\mathbf{M}_{E}$. Another proof of irreducibility of the image ${ }^{n} \mathbf{M}_{E}$ of $\cup D_{c}$ in $\mathbf{M}$ follows easily from Theorem 5.4 .5 of [8], which shows that ${ }^{n} \mathbf{M}_{E}$ $=P\left(\mathbf{M}_{E}^{s}\right)$ in the notation of [10], Sect. 2. Now, if $U$ is a connected component of the Hilbert scheme $\mathbf{H}_{E}^{d}$, then one can define the period mapping

$$
P: U \rightarrow \mathbf{M}_{E}
$$

which assigns to a polarized Enriques surface of degree $d$ in $\mathbb{P}^{n}$ the corresponding point in $D / C$. This map is a map of algebraic varieties, hence,

$$
U^{n}=P^{-1}\left({ }^{n} \mathbf{M}_{E}\right)
$$

is a closed Zariski subset in $U$. To prove that $U^{n} \neq U$ we can argue as follows. The group $S L(2 d+1)$ acts naturally on $\mathbf{H}_{E}^{d}$ leaving $U$ invariant (because the group is connected). The quotient space $U / S L(2 d+1)$ exists as an algebraic space $[17$, p. 54]. Its dimension is equal to 10 by Lemma 3.3. Obviously, the period mapping factors through the quotient and defines a map of algebraic spaces

$$
\bar{P}: U / S L(2 d+1) \rightarrow \mathbf{M}_{E} \text {. }
$$

Since the Picard group of an Enriques surface is discrete, there are only countably many projective isomorphism classes of polarized Enriques surfaces in any isomorphism class of Enriques surfaces. This shows that the fibres of $\bar{P}$ are discrete, hence finite, and $\bar{P}$ is a generically surjective map of 10-dimensional algebraic spaces. This proves that $P$ is generically surjective. Hence, $U \neq U^{n}$.

\section{Enriques Surfaces of Degree 10 in $\mathbb{P}^{5}$}

As follows from Corollary 2.6, every such surface is either unnodal or contains a smooth rational curve of degree at most 10 . In this section we will prove that, in fact, a nodal Enriques surface of degree 10 in $\mathbb{P}^{5}$ contains a smooth rational curve of degree less or equal to 4 .

Proposition 4.1. An Enriques surface cannot be embedded into $\mathbb{P}^{n}$ as a surface of degree less than 10 . If $S$ is an Enriques surface of degree 10 in $\mathbb{P}^{n}$ not lying in a proper subspace and $\operatorname{char}(k) \neq 2$, then $n=5$. 
Proof. Let $\phi=\phi_{H_{S}}$. It follows from the proof of Corollary 2.6, that $\phi(h) \geqq 3$ for the class $h$ of a very ample divisor on $S$. Fix an isometry $\sigma: H_{S} \rightarrow T_{2,3.7}$. Applying transformations from the Weyl group $W$, we may assume that $\sigma(h)$ belongs to the fundamental chamber, i.e. can be written as

$$
\sigma(h)=a_{0} \omega_{0}+\ldots+a_{9} \omega_{9},
$$

where $a_{i}$ are nonnegative integers and the $\omega_{i}$ 's are defined in Lemma 1.4. It is directly verified that if $h^{2}=8$, then

$$
\sigma(h)=\omega_{1}+\omega_{9}, 2 \omega_{8}, \text { or } \omega_{8}+3 \omega_{9} .
$$

However, since $\omega_{9}$ is an isotropic vector, we find that $\phi(\sigma(h)) \leqq 2$. The same computation can be made for the cases $h^{2} \leqq 8$. However, if $\operatorname{char}(k) \neq 2$, these cases can be excluded by other reason. Indeed, it is known that for any ample divisor $H$ on $S, H^{1}\left(S, \mathcal{O}_{S}(H)\right)=0$ (cf. Remark 3.2). Thus, $\operatorname{dim} H^{0}\left(S, \mathcal{O}_{S}(H)\right)=\frac{1}{2} H^{2}+1$. Obviously, $S$ cannot be a nonsingular sextic surface in $\mathbb{P}^{3}$. Also, we see why the last statement of the proposition is true.

Proposition 4.2. Let $S$ be an Enriques surface of degree 10 in $\mathbb{P}^{5}$. Then there exists an isometry $\sigma: H_{S} \rightarrow T_{2,3,7}$ such that $\sigma(h)=\Delta$, where $h$ is the class of a hyperplane section and $\Delta=\omega_{0}$, as defined in Lemma 1.4.

Proof. In the notation of the proof of the previous proposition, we may assume that $\sigma(h)=a_{0} \omega_{0}+\ldots+a_{9} \omega_{9}$, where

$$
\left(a_{0} \omega_{0}+\ldots+a_{9} \omega_{9}\right)^{2}=10 .
$$

By direct computation, we find that

$$
\sigma(h)=\omega_{0}, \omega_{7}+\omega_{9}, \quad \text { or } \omega_{8}+4 \omega_{9} .
$$

Only in the first case, $\phi(h) \geqq 3$ (in fact, $=3$ ), the condition which is necessary for very ampleness.

Remark 4.3. It is proven in [8] that every Enriques surface admits a birational morphism onto a surface of degree 10 in $\mathbb{P}^{5}$ with at most double rational singularities. In particular, every unnodal surface can be embedded into $\mathbb{P}^{5}$ as a surface of degree $10(\operatorname{char}(k) \neq 2)$.

Theorem 4.4. Let $S$ be a nodal Enriques surface of degree 10 in a projective space $\mathbb{P}^{n}$. Then $S$ contains a smooth rational curve of degree $\leqq 4$.

Proof. As before, we fix an isometry between $H_{S}$ and the lattice $T_{2,3,7}$ which sends the class $h$ of a hyperplane section of $S$ to the vector $\Delta$. Let $f$ be an isotropic vector in $H_{S}$. We call it irreducible if it represents an effective divisor $D$ on $S$ such that $|2 D|$ is an irreducible elliptic or quasi-elliptic pencil with no reducible fibres. Applying Lemma 1.4, we can write

$$
3 h=f_{1}+\ldots+f_{10},
$$

where $f_{i}$ are isotropic vectors with $f_{i} \cdot f_{j}=1, i \neq j, h \cdot f_{i}=3$. Let $f_{i j}=h-f_{i}-f_{j}, i \neq j$. These are isotropic vectors with $h \cdot f_{i j}=4$. 
Assume now that $S$ does not contain smooth rational curves of degree $\leqq 4$, i.e. for every class $\alpha$ of such curve $\alpha \cdot h>4$. Then, taking an effective representative of the vectors $f_{i}$ or $f_{i j}$, we see that they cannot contain smooth rational components. Thus, the vectors $f_{i}$ and $f_{i j}$ are irreducible.

Let us show that for every $s$ from the Weyl group $W$ of $H_{S}, s(h)$ is the class of an ample divisor and for every class $\alpha$ of a smooth rational curve $s(h) \cdot \alpha>4$. The proof is by induction on the length $\lg (s)$ of $s$ as a word in simple reflections $s_{i}=s_{\alpha_{i}}$, where $\alpha_{i}=f_{i}-f_{j}, j \neq 0$ and $\alpha_{0}=h-f_{1}-f_{2}-f_{3}$.

Clearly, $h$ is not changed after applying $s_{i}, i \neq 0$. If $s=s_{0}$,

$$
3 s(h)=f_{2,3}+f_{1,3}+f_{1,2}+f_{4}+\ldots+f_{10}=s\left(f_{1}\right)+\ldots+s\left(f_{10}\right) .
$$

Since all the summands are irreducible vectors, $s(h)$ intersects positively every effective class. Hence, it is the class of an ample divisor. Let $\alpha \in R^{+}\left(H_{S}\right)$ such that $s(h) \cdot \alpha \leqq 4$. We know that $\alpha$ intersects every irreducible vector positively, thus, $3 s(h) \cdot \alpha \geqq 10$, i.e. $s(h) \cdot \alpha=4$.

There are two possibilities: either $\alpha$ intersects one $s\left(f_{i}\right)$ at 3 and others at 1 , or $\alpha$ intersects two $s\left(f_{i}\right)$ 's at 2 and the remaining ones at 1 . If $\alpha \cdot s\left(f_{i}\right)=3$, then

$$
\alpha=s(h)-2 s\left(f_{i}\right) \text {. }
$$

This is seen by comparing the intersection of the both sides with the vectors $s\left(f_{i}\right)$. If $i>3$, then $s\left(f_{i}\right)=f_{i}$ and we easily get that $\alpha \cdot f_{1, i}=s(h) \cdot f_{1, i}-2 f_{i} \cdot f_{1, i}=0$. This contradicts the irreducibility of the vector $f_{1, i}$. If $i<3$, say $s\left(f_{i}\right)=f_{1,2}$, then we repeat the argument by taking $f_{2}$ instead of $f_{1, i}$.

Assume that $\alpha \cdot s\left(f_{i}\right)=\alpha \cdot s\left(f_{j}\right)=2$ for some $i \neq j$. Then

$$
\alpha=s(h)-s\left(f_{i}\right)-s\left(f_{j}\right),
$$

again, by comparing the intersections with the $s\left(f_{i}\right)$ 's. However, this implies that $\alpha=s\left(h-f_{i}-f_{j}\right)=s\left(f_{i, j}\right)$ is an isotropic vector.

If $s=s_{i} \circ s^{\prime}$, where $\lg \left(s^{\prime}\right)<\lg (s)$, then replacing $h$ by $s^{\prime}(h)$ and repeating the argument by using the induction, we obtain that $s(h)$ is the class of an ample divisor and $s(h) \cdot \alpha>4$ for any $\alpha \in R^{+}\left(H_{s}\right)$. Taking $s=s_{\alpha}$, we get $s(h) \cdot \alpha=h \cdot s(\alpha)=-h \cdot \alpha<0$ which is absurd.

Remark 4.5. Another proof of Theorem 4.5 proceeds as follows. One can directly compute the coordinates of roots $\alpha \in T_{2,3,7}$ with respect to the basis $e_{0}, \ldots, e_{10}$ for which $4<\alpha \cdot \Delta \leqq 10$. This is done by solving the diophantine equations

$$
\begin{aligned}
m_{0}^{2}-m_{1}^{2}-\ldots-m_{10}^{2} & =-2 \\
3 m_{0}-m_{1}-\ldots-m_{10} & =0
\end{aligned}
$$

with nonnegative $m_{i}$ 's, $i \neq 0$, and $4<m_{0} \leqq 10$. Then, one checks that for each such root there exists an isotropic vector $f$ with $0<f \cdot \alpha<m_{0}, f \cdot \alpha=0$. After this, the argument from the proof of Theorem 2.5 shows, that we can always replace a smooth rational curve on $S$ of degree $>4$ by a curve of smaller degree.

Remark 4.6. Let $V$ be a rational surface obtained by blowing up 10 points on the projective plane $\mathbb{P}^{2}$. Then

$$
\operatorname{Pic}(V)=\mathbb{Z} e_{0} \oplus \ldots \oplus \mathbb{Z} e_{10},
$$


where $e_{0}$ is the class of the inverse transform of a line in $\mathbb{P}^{2}$ and $e_{i}$ is the class of an exceptional curve blown up from one of the ten points. Since $K_{V}=-3 e_{0}$ $+e_{1}+\ldots+e_{10}$, the orthogonal complement of $K_{V}$ is isomorphic to the lattice $T_{2,3,7}$. Thus, every nodal curve on $V$, i.e. a smooth rational curve $E$ with $E \cdot K_{V}=0$, defines a root in $T_{2,3,7}$. The projection of such a curve to $\mathbb{P}^{2}$ is a plane irreducible curve of degree $m_{0}$ with $m_{i}$-multiple points at the ten points which we have blown up. The numbers $m_{0}, \ldots, m_{10}$ satisfy the diophantine equations from Remark 4.7. The existence of such a curve is a "discriminant condition" on the ten points $[6,9]$. In general, it is impossible to lower the degree $m_{0}$ of a discriminant condition. However, if we additionally assume that $\left|-2 K_{V}\right|$ is non-empty and represented by an irreducible curve (a plane sextic with 10 double points), then we can obtain analogues of all results of this paper. For example, we can prove that every discriminant condition reduces to a condition of degree at most 4 . The reason of imposing the above condition on $\left|-2 K_{V}\right|$ is simple. In this case one can prove an analogue of Lemma 2.4 [where $H_{S}$ is replaced by $\left(K_{V}\right)_{\text {Pic } V}^{\perp}$ ] and repeat the argument of the proof of Theorem 2.5.

Note that the surfaces $V$ from above (Coble surfaces) can be realized as certain degenerations of Enriques surfaces. They can be birationally mapped onto a surface of degree 10 in $\mathbb{P}^{5}$ with double rational singularities and one quadruple point.

The reduction of discriminant conditions on Coble surfaces to the conditions of degree at most 4 was stated with a wrong proof by Coble [7]. The right idea of the proof belongs to Hilda Hudson [12] whose proof is incomplete also.

Remark 4.7. It is easy to see that any root in $L=T_{2,3,7}$ is equivalent modulo $2 L$ to one of the following $496\left(=2^{4}\left(2^{5}-1\right)\right)$ roots:

$$
\begin{aligned}
& \left(\begin{array}{c}
10 \\
2
\end{array}\right) \text { of type } e_{i}-e_{j}, \\
& \left(\begin{array}{c}
10 \\
3
\end{array}\right) \text { of type } e_{0}-e_{i}-e_{j}-e_{k}, \\
& \left(\begin{array}{c}
10 \\
4
\end{array}\right) \text { of type } 2 e_{0}-e_{i}-e_{j}-e_{k}-e_{m}-e_{n}-e_{r}, \\
& \left(\begin{array}{c}
10 \\
3
\end{array}\right) \text { of type } 3 e_{0}-2 e_{i}-e_{j}-e_{k}-e_{m}-e_{n}-e_{t}-e_{r}-e_{s}, \\
& 1 \text { of type } 4 e_{0}-3 e_{1}-e_{2}-\ldots-e_{10},
\end{aligned}
$$

where all indices are distinct.

Let us fix a divisor $H$ on an Enriques surface $S$ such that the linear system $|H|$ defines a birational morphism onto a surface of degree 10 with at most double rational singularities (cf. Remark 4.3). Let $h$ be the class of $H$ in $H_{S}$ and $\sigma: H_{S} \rightarrow L$ be an isometry which maps $h$ to the class $\Delta$. It follows from Theorem 4.6 that $S$ contains smooth rational curves whose classes are represented by a vector $m_{0} e_{0}-m_{1} e_{1}-\ldots-m_{10} e_{10}$ in $L_{0}$ with $m_{0} \leqq 4$. Solving the corresponding diophantine equations (cf. Remark 4.5), we find that such a vector must be one of the 496 vectors above or equal to a vector $\alpha=4 e_{0}-2 e_{i}-2 e_{j}-2 e_{k}-e_{m}-e_{n}-e_{t}-e_{r}$ $-e_{s}-e_{p}$. However, the latter type can be reduced to the vector $\alpha^{\prime}=2 e_{0}-e_{m}-e_{n}$ 
$-e_{t}-e_{r}-e_{s}-e_{p}$ by the algorithm of the proof of Theorem 2.5. To see this, one considers the isotropic vector $f=3 e_{0}-e_{i}-e_{j}-e_{k}-e_{m}-e_{n}-e_{r}-e_{t}-e_{s}-e_{p}$ and notice that $f \cdot \alpha=0,2 f-\alpha=\alpha^{\prime}$.

Observe that the curves of the first type represent the curves blown down to double rational points by the map given by the linear system $|H|$. The curves of the remaining types represent lines, conics, cubic and quartic rational curves on the image.

For a "generic" nodal rational surface $S$ any smooth rational curve can be reduced by an automorphism of $S$ to a curve of one of the 496 types and this class depends only on the choice of $h$. Here, a generic nodal surface can be defined as a nodal surface which admits an embedding into $\mathbb{P}^{5}$ as a surface of degree 10 lying on a nonsingular quadric and not containing smooth rational curves of degree less than 4. This condition implies that $S$ is isomorphic to a Reye congruence of lines in $\mathbb{P}^{3}$. The proof of the above result will be published in a future paper.

Acknowledgements. We owe much to stimulating discussions on the subject of Enriques surfaces with W. Barth, E. Looijenga, S. Mukai, Y. Namikawa, and C. Peters.

\section{References}

1. Artin, M.: Algebrization of formal moduli. I. Collection of Math. Papers in Honot of K. Kodaira, pp. 21-71. Tokyo: Univ. of Tokyo Press 1970

2. Artin, M.: Versal deformations and algebraic stacks. Invent. Math. 27, 165-189 (1974)

3. Barth, W., Peters, C.: Automorphisms of Enriques surfaces. Invent. Math. 73, 383-411 (1983)

4. Bombieri, E., Mumford, D.: Enriques' classification of surfaces in char $p$. II. Collection of Papers dedicated to K. Kodaira, pp. 23-42. Cambridge: Cambridge University Press 1977

5. Bourbaki, N.: Groupes et algèbres de Lie. Chaps. 4-6. Paris: Hermann 1968

6. Coble, A.: Algebraic geometry and theta functions. Amer. Math. Soc. Coll. Publ., Vol. 10. Providence 1929

7. Coble, A.: The ten nodes of the rational sextic and of the Cayley symmetroid. Am. J. Math. 41, 243-265 (1919)

8. Cossec, F.: On the Picard group of Enriques surfaces. Math. Ann. (to appear)

9. Dolgachev, 1.: Weyl groups and Cremona transformations. Proc. Symp. Pure Math., Am. Math. Soc. 40, Part 1, 283-294 (1983)

10. Dolgachev, I.: On automorphisms of Enriques surfaces. Invent Math. 76, 163-177 (1984)

11. Horikawa, E.: On the periods of Enriques surfaces. I. Math. Ann. 234, 73-108 (1978)

12. Hudson, $H$.: The Cremona transformations of a certain plane sextic. Proc. London Math. Soc., Ser. 2 15, 385-403 (1916/17)

13. Lang, W.: On Enriques surfaces in characteristic p. I. Math. Ann. 265, 45-65 (1983)

14. Mumford, D.: Enriques' classification of surfaces in char p. I. In: Global analysis, pp. 325-339. Princeton: Princeton University Press 1969

15. Namikawa, Y.: Periods of Enriques surfaces (preprint)

16. Piatecky-Shapiro, I., Shafarevich, I.: A Torelli theorem for algebraic surfaces of type K3. Izv. Akad. Nauk SSSR, Ser. Math. 35, 530-572 (1971) [Engl. Transl.: Math. USSR-Izv. 5, 547-588 (1971)]

17. Popp, H.: Moduli theory and classification theory of algebraic varieties. Lect. Notes Math. 620. Berlin, Heidelberg, New York: Springer 1977

18. Vinberg, E.: Some discrete groups in Lobacevskii spaces. In: Discrete subgroups of Lie groups, pp. 323-348. Oxford: Oxford University Press 1975 